\title{
Least Squares Approximation With Constraints
}

\section{By Gradimir V. Milovanović and Staffan Wrigge}

\begin{abstract}
In this paper we study two families of functions $F_{e}$ and $F_{o}$, and show how to approximate the functions in the interval $[-1,1]$. The functions are assumed to be real when the argument is real. We define
\end{abstract}

$$
F_{e}=\left\{f: f(-x)=f(x), f(1)=0, f \in L^{2}[-1,1]\right\}
$$

and

$$
F_{o}=\left\{f: f(-x)=-f(x), f(1)=0, f \in L^{2}[-1,1]\right\} .
$$

Let further $\mathscr{P}_{m}$ be the set of all real polynomials of degree not higher than $m$ such that the polynomials belong to the set $F_{e}$ if $m$ is even and to the set $F_{o}$ if $m$ is odd.

We determine the least squares approximation for the function $f \in F_{e}$ (or $F_{o}$ ) in the class $\mathscr{P}_{2 n}$ (or $\mathscr{P}_{2 n+1}$ ), with respect to the norm $\|f\|=((f, f))^{1 / 2}$, where the inner product is defined by $(f, g)=\int_{-1}^{1} f(x) g(x) w(x) d x$, with $f, g \in L^{2}[-1,1]=L_{w}^{2}[-1,1]$ and $w(x)=$ $\left(1-x^{2}\right)^{\lambda-1 / 2}$.

We also consider the general case when $f$ is neither an even nor an odd function but $f \in L^{2}[-1,1]$ and $f(-1)=f(1)=0$.

Using the theory of Gegenbauer polynomials we obtain the approximating polynomials in the form

$$
\phi_{2 n}(x)=\sum_{k=1}^{n} d_{n, k}\left(1-x^{2}\right)^{k} \quad \text { when } f \in F_{e}
$$

and

$$
\phi_{2 n+1}(x)=x \sum_{k=1}^{n} e_{n, k}\left(1-x^{2}\right)^{k} \quad \text { when } f \in F_{o} .
$$

We apply the general theory to the functions $f(x)=\cos (\pi x / 2)$ and $f(x)=J_{0}\left(a_{0} x\right)$, where $a_{0}=\left\{\min x>0: J_{0}(x)=0\right\}$.

0. Introduction. In [12] Wrigge and Fransén considered two families of functions, viz., $F$ and $H$, and showed how these functions can be approximated on $[0,1]$ by polynomials of the form

$$
\sum_{n=1}^{k} c_{n, k}(x(1-x))^{n} \text { and }(1-2 x) \sum_{n=1}^{k} C_{n, k}(x(1-x))^{n} .
$$

They used the $L_{2}$-norm with respect to the weight function $w(x)=(x(1-x))^{q}$, where $q \in\{0,1,2, \ldots\}$. This method of approximation can be further generalized, as was shown in Wrigge [11], by using Bernstein polynomials.

Received December 13, 1983; revised November 13, 1984 and June 3, 1985.

1980 Mathematics Subject Classification. Primary 41A10; Secondary 33A45, 65D15, 65D20.

Key words and phrases. Approximation theory, Gegenbauer polynomials. 
However, a better and more natural way is to use Gegenbauer polynomials $C_{k, \lambda}(x)$ orthogonal with respect to the weight function $w(x)=\left(1-x^{2}\right)^{\lambda-1 / 2}$, $x \in[-1,1], \lambda>-1 / 2$.

The families of functions to be approximated are denoted by $F_{e}$ and $F_{o}$ (defined in the abstract) which are natural extensions of $F$ and $H$ used by Wrigge and Fransén [12]. The advantage of our new method is that we can avoid the rather cumbersome manipulations with matrices which were found necessary in the work of Wrigge and Fransén.

1. Preliminaries and Definitions. We define the Gegenbauer polynomials $\left\{C_{n, \lambda}(x)\right\}$ by means of the generating function

$$
\left(1-2 x t+t^{2}\right)^{-\lambda}=\sum_{n=0}^{\infty} C_{n, \lambda}(x) t^{n}, \quad \lambda \neq 0 .
$$

Using this generating function and some manipulations we may prove the recurrence relations

$$
\begin{gathered}
(k+1) C_{k+1, \lambda}(x)=2(k+\lambda) x C_{k, \lambda}(x)-(k+2 \lambda-1) C_{k-1, \lambda}(x) \\
(k+\lambda) C_{k, \lambda}(x)=\lambda C_{k, \lambda+1}(x)-\lambda C_{k-2, \lambda+1}(x) \\
\begin{aligned}
2 \lambda\left(1-x^{2}\right) C_{k, \lambda+1}(x) & =(2 \lambda+k) C_{k, \lambda}(x)-(k+1) x C_{k+1, \lambda}(x) \\
& =(k+1+2 \lambda) x C_{k+1, \lambda}(x)-(k+2) C_{k+2, \lambda}(x) .
\end{aligned}
\end{gathered}
$$

Starting values are given by $C_{0, \lambda}(x)=1, C_{1, \lambda}(x)=2 \lambda x$.

In the sequel we will also need the formulae

$$
C_{k, \lambda}(1)=\left(\begin{array}{c}
k+2 \lambda-1 \\
k
\end{array}\right)=\frac{(2 \lambda)_{k}}{k !} \text { and } C_{k, \lambda}(-x)=(-1)^{k} C_{k, \lambda}(x) .
$$
81])

Of interest is the limit behavior of $C_{k, \lambda}(x)$ when $\lambda \rightarrow 0$, given by (see, e.g., [10, p.

$$
\lim _{\lambda \rightarrow 0} \frac{C_{k, \lambda}(x)}{\lambda}=\frac{2}{k} T_{k}(x), \quad k=1,2, \ldots,
$$

where $T_{k}(x)$ denotes the Chebyshev polynomial of the first kind.

More important to us are the following two relations, since they enable us to write the approximating polynomials in a form similar to that used by Wrigge and Fransén [12],

$$
\begin{gathered}
C_{2 k, \lambda}(x)=C_{2 k, \lambda}(1){ }_{2} F_{1}\left(-k, k+\lambda ; \lambda+\frac{1}{2} ; 1-x^{2}\right), \\
C_{2 k+1, \lambda}(x)=C_{2 k+1, \lambda}(1) x_{2} F_{1}\left(-k, k+\lambda+1 ; \lambda+\frac{1}{2} ; 1-x^{2}\right)
\end{gathered}
$$

(see, e.g., Erdélyi et al. [3, p. 176]).

We next prove the following auxiliary result (see also a paper by Rakovich and Vasić [7]).

Lemma. Let

$$
h_{k}=\left\|C_{k, \lambda}\right\|^{2}=\frac{\lambda}{(k+\lambda) \Lambda(\lambda)} C_{k, \lambda}(1), \quad \Lambda(\lambda)=\frac{\Gamma(\lambda+1)}{\sqrt{\pi} \Gamma\left(\lambda+\frac{1}{2}\right)} .
$$


Then the identities

$$
S_{0}(x)=\sum_{k=0}^{n} \frac{C_{2 k, \lambda}(x) C_{2 k, \lambda}(1)}{h_{2 k}}=\Lambda(\lambda) C_{2 n, \lambda+1}(x)
$$

and

$$
S_{1}(x)=\sum_{k=0}^{n} \frac{C_{2 k+1, \lambda}(x) C_{2 k+1, \lambda}(1)}{h_{2 k+1}}=\Lambda(\lambda) C_{2 n+1, \lambda+1}(x)
$$

hold.

Proof. We could use the Christoffel-Darboux identity

$$
\sum_{k=0}^{m} \frac{C_{k, \lambda}(x) C_{k, \lambda}(t)}{h_{k}}=q_{m} \frac{C_{m+1, \lambda}(x) C_{m, \lambda}(t)-C_{m, \lambda}(x) C_{m+1, \lambda}(t)}{x-t},
$$

where $q_{m}=(m+1) ! \Lambda(\lambda) / 2 \lambda(2 \lambda)_{m}$ (see, e.g., [1, p.785]) in combination with (1.2). However, there exists a simpler proof based on induction. The statement of the lemma indeed may be written in the form

$$
C_{2 n, \lambda+1}(x)=\sum_{k=0}^{n} \frac{2 k+\lambda}{\lambda} C_{2 k, \lambda}(x)
$$

and

$$
C_{2 n+1, \lambda+1}(x)=\sum_{k=0}^{n} \frac{2 k+1+\lambda}{\lambda} C_{2 k+1, \lambda}(x) .
$$

Note that (1.6) is correct for $n=1$. From (1.1) we find

$$
C_{2 n+2, \lambda+1}(x)=C_{2 n, \lambda+1}(x)+\frac{2 n+2+\lambda}{\lambda} C_{2 n+2, \lambda}(x)
$$

showing that (1.6) holds true also for $n:=n+1$. Equation (1.7) is proved similarly.

Now let the functions $f, g \in L^{2}[-1,1]=L_{w}^{2}[-1,1]$ and denote by

$$
(f, g)=\int_{-1}^{1} f(x) g(x) w(x) d x
$$

the inner product of $f$ and $g$, where $w(x)=\left(1-x^{2}\right)^{\lambda-1 / 2}, \lambda>-1 / 2$.

We introduce two families of real functions, viz.

$$
F_{e}=\left\{f: f(-x)=f(x), f(1)=0, f \in L^{2}[-1,1]\right\}
$$

and

$$
F_{o}=\left\{f: f(-x)=-f(x), f(1)=0, f \in L^{2}[-1,1]\right\} .
$$

Let further $\mathscr{P}_{m}$ be the set of all real polynomials of degree not higher than $m$ and such that the polynomials belong to the set $F_{e}$ if $m$ is even and to the set $F_{o}$ if $m$ is odd.

We will determine the least squares approximation $\phi_{2 n}\left(\right.$ or $\left.\phi_{2 n+1}\right)$ for the function $f \in F_{e}\left(\right.$ or $\left.F_{o}\right)$ in the class $\mathscr{P}_{2 n}\left(\right.$ or $\left.\mathscr{P}_{2 n+1}\right)$, with respect to the norm $\|f\|=((f, f))^{1 / 2}$, where the inner product is defined by (1.8). For this approximation we have

$$
\min _{\phi \in \mathscr{P}_{2 n}}\|f-\phi\|=\left\|f-\phi_{2 n}\right\| \quad \text { when } f \in F_{e},
$$


or

$$
\min _{\phi \in \mathscr{P}_{2 n+1}}\|f-\phi\|=\left\|f-\phi_{2 n+1}\right\| \quad \text { when } f \in F_{o} .
$$

In the general case, when $f$ is neither an even nor an odd function, but $f \in L^{2}[-1,1]$ and $f(-1)=f(1)=0$, then the least squares approximation $\psi_{m}$ (in the class of real polynomials of degree $\leqslant m$ ), which satisfies the conditions $\psi_{m}(-1)=\psi_{m}(1)=0$, is simply

$$
\psi_{m}(x)=\phi_{2 n}(x)+\phi_{2 n+1}(x) \text { when } m=2 n+1
$$

and

$$
\psi_{m}(x)=\phi_{2 n}(x)+\phi_{2 n-1}(x) \text { when } m=2 n,
$$

where $\phi_{2 n}$ and $\phi_{2 n \mp 1}$ are the solutions of (1.9) and (1.10). This can be seen by writing

$$
f(x)=\frac{1}{2}(f(x)+f(-x))+\frac{1}{2}(f(x)-f(-x)) .
$$

Our main results are Theorems 1 and 2 of Section 2. Section 3 treats more general constraints. Examples are given in Section 4.

\section{Approximations With Simple Constraints.}

THEOREM 1. If $f \in F_{e}$, then the least squares approximation in the class $\mathscr{P}_{2 n}$ is given by

$$
\phi_{2 n}(x)=\Lambda(\lambda) \sum_{k=1}^{n} a_{n, k}(\lambda)\left(1-x^{2}\right)^{k}
$$

where

$$
a_{n, k}(\lambda)=\frac{(-1)^{k}}{\left(\lambda+\frac{1}{2}\right)_{k+1}} \sum_{m=0}^{n} \frac{2 m+\lambda}{\lambda}\left(f, C_{2 m, \lambda}\right) \alpha_{m, k}^{(n)}(\lambda) \quad(\lambda \neq 0)
$$

and

$$
\alpha_{m, k}^{(n)}(\lambda)= \begin{cases}-\left(\begin{array}{l}
n \\
k
\end{array}\right)(n+\lambda+1)_{k}\left(\lambda+\frac{1}{2}\right), & m<k, \\
\left(\begin{array}{c}
m \\
k
\end{array}\right)(m+\lambda)_{k}\left(\lambda+k+\frac{1}{2}\right)-\left(\begin{array}{l}
n \\
k
\end{array}\right)(n+\lambda+1)_{k}\left(\lambda+\frac{1}{2}\right), & m \geqslant k\end{cases}
$$

When $\lambda=0$ we have

$$
a_{n, k}(0)=\frac{(-1)^{k}}{\left(\frac{1}{2}\right)_{k+1}} \theta_{n} \sum_{m=0}^{n}\left(f, T_{2 m}\right) \alpha_{m, k}^{(n)}(0),
$$

where $\theta_{0}=1$ and $\theta_{n}=2$, when $n \geqslant 1$.

Note 1. The approximation with constraint $\phi_{2 n}(x)$ turns out to be the truncated expansion in Gegenbauer polynomials with a multiple of $S_{0}(x)\left(=\Lambda(\lambda) C_{2 n, \lambda+1}(x)\right)$ added to satisfy the constraint at $x=1$, i.e.,

$$
\phi_{2 n}(x)=\tilde{\phi}_{2 n}(x)-\tilde{\phi}_{2 n}(1) \frac{S_{0}(x)}{S_{0}(1)}
$$


where $\tilde{\phi}_{2 n}(x)$ is the least squares approximation without constraint given by (see, e.g., Rivlin [8, pp. 50-51])

$$
\tilde{\phi}_{2 n}(x)=\sum_{k=0}^{n} \frac{\left(f, C_{2 k, \lambda}\right)}{h_{2 k}} C_{2 k, \lambda}(x) \text {. }
$$

Proof of Theorem 1. Let $f \in F_{e}$ and $\lambda \neq 0$. In order to find the minimum of the "distance" $\left\|f-\phi_{2 n}\right\|$ under the constraint $\phi_{2 n}(1)=0$, we represent $\phi_{2 n}$ as a linear combination of the Gegenbauer polynomials $\left\{C_{2 k, \lambda}(x)\right\}$ and consider the associated function $D_{e}$, viz.

$$
D_{e}=\int_{-1}^{1}\left(f(x)-\sum_{k=0}^{n} d_{k} C_{2 k, \lambda}(x)\right)^{2} w(x) d x+\mu \sum_{k=0}^{n} d_{k} C_{2 k, \lambda}(1),
$$

where $\mu$ is a Lagrange multiplier, whose value we have to determine. We must have

$$
\frac{\partial D_{e}}{\partial d_{i}}=-2\left(f, C_{2 i, \lambda}\right)+2 h_{2 i} d_{i}+\mu C_{2 i, \lambda}(1)=0, \quad i=0,1, \ldots, n,
$$

which yields

$$
d_{i}=\frac{1}{h_{2 i}}\left(\left(f, C_{2 i, \lambda}\right)-\frac{1}{2} \mu C_{2 i, \lambda}(1)\right), \quad i=0,1, \ldots, n .
$$

From the constraint $\phi_{2 n}(1)=0$ we find

$$
\frac{1}{2} \mu=\sum_{k=0}^{n} \frac{\left(f, C_{2 k, \lambda}\right)}{h_{2 k}} C_{2 k, \lambda}(1) / \sum_{k=0}^{n} \frac{C_{2 k, \lambda}^{2}(1)}{h_{2 k}}=\frac{\tilde{\phi}_{2 n}(1)}{S_{0}(1)},
$$

where $\tilde{\phi}_{2 n}$ is given by (2.2) and $S_{0}$ by the lemma. Now, we note that the formula (2.1) follows from (2.2), (2.3) and (2.4).

Using the representation of Gegenbauer polynomials in terms of hypergeometric functions (Eqs. (1.3) and (1.4)) we get

$$
\tilde{\phi}_{2 n}(x)=\sum_{m=0}^{n} \frac{\left(f, C_{2 m, \lambda}\right)}{h_{2 m}} C_{2 m, \lambda}(1){ }_{2} F_{1}\left(-m, m+\lambda ; \lambda+\frac{1}{2} ; 1-x^{2}\right)
$$

and

$$
S_{0}(x)=S_{0}(1){ }_{2} F_{1}\left(-n, n+\lambda+1 ; \lambda+\frac{3}{2} ; 1-x^{2}\right) .
$$

A known formula for the hypergeometric function yields

$$
\begin{aligned}
\tilde{\phi}_{2 n}(x) & =\Lambda(\lambda) \sum_{m=0}^{n} \frac{2 m+\lambda}{\lambda}\left(f, C_{2 m, \lambda}\right) \sum_{k=0}^{m}(-1)^{k}\left(\begin{array}{c}
m \\
k
\end{array}\right) \frac{(m+\lambda)_{k}}{\left(\lambda+\frac{1}{2}\right)_{k}}\left(1-x^{2}\right)^{k} \\
& =\Lambda(\lambda) \sum_{k=0}^{n} \frac{(-1)^{k}\left(1-x^{2}\right)^{k}}{\left(\lambda+\frac{1}{2}\right)_{k}} \sum_{m=k}^{n} \frac{2 m+\lambda}{\lambda}\left(f, C_{2 m, \lambda}\right)\left(\begin{array}{c}
m \\
k
\end{array}\right)(m+\lambda)_{k}
\end{aligned}
$$

and

$$
\frac{S_{0}(x)}{S_{0}(1)}=\sum_{k=0}^{n}(-1)^{k}\left(\begin{array}{l}
n \\
k
\end{array}\right) \frac{(n+\lambda+1)_{k}}{\left(\lambda+\frac{3}{2}\right)_{k}}\left(1-x^{2}\right)^{k} .
$$

On the basis of the above and the formula (2.1), we obtain the assertion of Theorem 1 for $\lambda \neq 0$. We note that $a_{n, 0}(\lambda)=0$. The case $\lambda=0$ is handled by means of a limit process. 
It is of some interest to compare approximations without constraints with our approximations with constraints. First of all, we note that $\phi_{2 n}\left(x_{k}\right)=\tilde{\phi}_{2 n}\left(x_{k}\right)$, where $x_{k}$ is any zero of the polynomial $C_{2 n, \lambda+1}$. Assuming $f \in F_{e}$, define

$$
D_{e}^{*}=\min _{\phi \in \mathscr{P}_{2 n}}\|f-\phi\|^{2}=\left\|f-\phi_{2 n}\right\|^{2}
$$

and

$$
\tilde{D}_{e}^{*}=\min _{\phi \in \pi_{2 n}}\|f-\phi\|^{2}=\left\|f-\tilde{\phi}_{2 n}\right\|^{2}
$$

where $\pi_{2 n}$ is the set of all real polynomials of degree less than or equal to $2 n$. We note that $\mathscr{P}_{2 n} \subset \pi_{2 n}$. Using standard calculations and the constraint $\phi_{2 n}(1)=0$, we obtain

$$
D_{e}^{*}=\left(f-\phi_{2 n}, f-\phi_{2 n}\right)=\|f\|^{2}-\sum_{k=0}^{n} d_{k}^{2} h_{2 k} \geqslant 0,
$$

where $d_{k}$ is given by (2.3) and (2.4). We also note that

$$
\begin{aligned}
\sum_{k=0}^{n} d_{k}^{2} h_{2 k} & =\sum_{k=0}^{n} d_{k}\left(f, C_{2 k, \lambda}\right) \\
& =\sum_{k=0}^{n}\left(\frac{\left(\left(f, C_{2 k, \lambda}\right)\right)^{2}}{h_{2 k}}-\frac{\mu}{2} \frac{C_{2 k, \lambda}(1)\left(f, C_{2 k, \lambda}\right)}{h_{2 k}}\right)
\end{aligned}
$$

Using the lemma and the value of $\mu$ from (2.4) we obtain

$$
\sum_{k=0}^{n} d_{k}^{2} h_{2 k}=\sum_{k=0}^{n} \frac{\left(\left(f, C_{2 k, \lambda}\right)\right)^{2}}{h_{2 k}}-\frac{\mu^{2}}{4} S_{0}(1)
$$

Similarly, we find

$$
\tilde{D}_{e}^{*}=\left(f-\tilde{\phi}_{2 n}, f-\tilde{\phi}_{2 n}\right)=\|f\|^{2}-\sum_{k=0}^{n} \frac{\left(\left(f, C_{2 k, \lambda}\right)\right)^{2}}{h_{2 k}} .
$$

Using the expressions for $D_{e}^{*}$ and $\tilde{D}_{e}^{*}$ we get

$$
D_{e}^{*}-\tilde{D}_{e}^{*}=\frac{\mu^{2}}{4} S_{0}(1)=\frac{\left(\left(f, S_{0}\right)\right)^{2}}{S_{0}(1)}=\frac{\left(\tilde{\phi}_{2 n}(1)\right)^{2}}{\Lambda(\lambda) C_{2 n, \lambda+1}(1)}
$$

since, by the lemma, $\tilde{\phi}_{2 n}(1)=\left(f, S_{0}\right)$. Thus we see that the difference $D_{e}^{*}-\tilde{D}_{e}^{*}$ is proportional to the square of the error $\tilde{\phi}_{2 n}(1)-f(1)=\tilde{\phi}_{2 n}(1)$.

In a similar way the following result can be proved.

THEOREM 2. If $f \in F_{o}$, then the least squares approximation in the class $\mathscr{P}_{2 n+1}$ is given by

$$
\phi_{2 n+1}(x)=\Lambda(\lambda) x \sum_{k=1}^{n} b_{n, k}(\lambda)\left(1-x^{2}\right)^{k}
$$

where

$$
b_{n, k}(\lambda)=\frac{(-1)^{k}}{\left(\lambda+\frac{1}{2}\right)_{k+1}} \sum_{m=0}^{n} \frac{2 m+\lambda+1}{\lambda}\left(f, C_{2 m+1, \lambda}\right) \beta_{m, k}^{(n)}(\lambda) \quad(\lambda \neq 0)
$$


and

$$
\beta_{m, k}^{(n)}(\lambda)=\left\{\begin{array}{lr}
-\left(\begin{array}{l}
n \\
k
\end{array}\right)(n+\lambda+2)_{k}\left(\lambda+\frac{1}{2}\right), & m<k, \\
\left(\begin{array}{c}
m \\
k
\end{array}\right)(m+\lambda+1)_{k}\left(\lambda+k+\frac{1}{2}\right)-\left(\begin{array}{l}
n \\
k
\end{array}\right)(n+\lambda+2)_{k}\left(\lambda+\frac{1}{2}\right), m \geqslant k .
\end{array}\right.
$$

When $\lambda=0$ we have

$$
b_{n, k}(0)=\frac{2(-1)^{k}}{\left(\frac{1}{2}\right)_{k+1}} \sum_{m=0}^{n}\left(f, T_{2 m+1}\right) \beta_{m, k}^{(n)}(0) .
$$

Note 2. The approximation with constraint $\phi_{2 n+1}(x)$ turns out to be the truncated expansion in Gegenbauer polynomials with a multiple of $S_{1}(x)\left(=\Lambda(\lambda) C_{2 n+1, \lambda+1}(x)\right)$ added to satisfy the constraint at $x=1$, i.e.,

$$
\phi_{2 n+1}(x)=\tilde{\phi}_{2 n+1}(x)-\tilde{\phi}_{2 n+1}(1) \frac{S_{1}(x)}{S_{1}(1)}
$$

where $\tilde{\phi}_{2 n+1}$ is the corresponding least squares approximation without constraint.

The approximation over the interval [0,1], which was treated in [12], may be obtained from Theorems 1 and 2, if we replace $x$ by $1-2 x$. The case $\lambda=q+\frac{1}{2}$, where $q \in\{0,1,2, \ldots\}$ was investigated by Wrigge and Fransén [12].

We have found it convenient to introduce two further notations, viz.

$$
d_{n, k}(\lambda)=\Lambda(\lambda) a_{n, k}(\lambda) \text { and } e_{n, k}(\lambda)=\Lambda(\lambda) b_{n, k}(\lambda) .
$$

Sometimes we will omit the variable $\lambda$ and write only $d_{n, k}$ and $e_{n, k}$. The approximating polynomials in Theorems 1 and 2 may then be written as

$$
\phi_{2 n}(x)=\sum_{k=1}^{n} d_{n, k}\left(1-x^{2}\right)^{k} \quad \text { and } \quad \phi_{2 n+1}(x)=x \sum_{k=1}^{n} e_{n, k}\left(1-x^{2}\right)^{k} .
$$

3. Approximations With More General Constraints. The approach of approximation developed in Section 2 clearly enables one to introduce more general constraints, such as $f(a)=\phi(a)$, or even more generally $L \phi=c=$ const, where $L$ is a linear functional.

Suppose, e.g., that $f \in F_{e}$; then we could study the problem

$$
\min _{\phi \in \mathscr{P}_{2 n}}\|f-\phi\| \text { subject to } \int_{-1}^{1} f(x) d x=\int_{-1}^{1} \phi(x) d x .
$$

This problem becomes very natural in case $f(x)$ is an even probability density function defined on $[-1,1]$. Then of course $\int_{-1}^{1} f(x) d x=1$.

We will here not go too much into details but will only give some examples to show the complications involved. We start with the "most natural" generalization of the problem dealt with in Section 2, viz.

Let $f \in F_{e}$, minimize $\left\|f-\phi_{2 n}\right\|$ subject to $\phi_{2 n} \in \mathscr{P}_{2 n}$ and $\phi_{2 n}(a)=f(a)$, where $a \in[0,1)$. 
Put as before $\phi_{2 n}=\sum_{k=0}^{n} d_{k} C_{2 k, \lambda}(x)$ and define

$$
\begin{aligned}
\Delta_{e}= & \int_{-1}^{1}\left(f(x)-\sum_{k=0}^{n} d_{k} C_{2 k, \lambda}(x)\right)^{2} w(x) d x+\mu \sum_{k=0}^{n} d_{k} C_{2 k, \lambda}(1) \\
& +\gamma\left\{\sum_{k=0}^{n} d_{k} C_{2 k, \lambda}(a)-f(a)\right\},
\end{aligned}
$$

where $\mu$ and $\gamma$ are Lagrange multipliers, whose values we will determine.

In order to minimize $\Delta_{e}$ we must have, for $i=0,1,2, \ldots, n$,

$$
\frac{\partial \Delta_{e}}{\partial d_{i}}=-2\left(f, C_{2 i, \lambda}\right)+2 h_{2 i} d_{i}+\mu C_{2 i, \lambda}(1)+\gamma C_{2 i, \lambda}(a)=0 .
$$

This yields

$$
d_{i}=\frac{\left(f, C_{2 i, \lambda}\right)}{h_{2 i}}-\frac{\mu}{2} \cdot \frac{C_{2 i, \lambda}(1)}{h_{2 i}}-\frac{\gamma}{2} \cdot \frac{C_{2 i, \lambda}(a)}{h_{2 i}}, \quad i=0,1,2, \ldots, n .
$$

The constraints $\phi_{2 n}(1)=0$ and $\phi_{2 n}(a)=f(a)$ yield the system of equations

$$
\begin{aligned}
& A(1,1) \frac{\mu}{2}+A(a, 1) \frac{\gamma}{2}=(f, A(1, x)), \\
& A(1, a) \frac{\mu}{2}+A(a, a) \frac{\gamma}{2}=(f, A(a, x))-f(a),
\end{aligned}
$$

where

$$
A(x, t)=\sum_{k=0}^{n} \frac{C_{2 k, \lambda}(x) C_{2 k, \lambda}(t)}{h_{2 k}} .
$$

We note that $A(x, t)=A(t, x)$.

Since in Section 4 (Example 4.3) we will approximate $f(x)=\cos (\pi x / 2)$ with the extra constraint $\phi_{2 n}(0)=1$, it is convenient to take a closer look at the sums occurring in (3.1).

From the lemma we conclude that $(f, A(1, x))=\Lambda(\lambda)\left(f, C_{2 n, \lambda+1}\right)$, and the Christoffel-Darboux identity [cf. (1.5)] yields

$$
A(x, t)=\frac{q_{2 n+1}}{x^{2}-t^{2}}\left|\begin{array}{ll}
t C_{2 n+1, \lambda}(t) & C_{2 n+2, \lambda}(t) \\
x C_{2 n+1, \lambda}(x) & C_{2 n+2, \lambda}(x)
\end{array}\right| .
$$

The case $x=t$ is handled by a limit process. Thus we get

$$
A(t, t)=\lambda q_{2 n+1}\left|\begin{array}{cc}
C_{2 n+1, \lambda+1}(t) & C_{2 n, \lambda+1}(t) \\
C_{2 n+2, \lambda}(t) & C_{2 n+1, \lambda}(t)
\end{array}\right|-q_{2 n+1} \frac{C_{2 n+2, \lambda}(t) C_{2 n+1, \lambda}(t)}{2 t} .
$$

For example, if $t=0$ we have

$$
A(x, 0)=Q \frac{C_{2 n, \lambda+1}(0)}{C_{2 n, \lambda+1}(1)} \cdot \frac{C_{2 n+1, \lambda}(x)}{2 \lambda x} \text { and } A(0,0)=Q \frac{C_{2 n, \lambda+1}^{2}(0)}{C_{2 n, \lambda+1}(1)},
$$

where $Q=(2 n+1)(2 \lambda+2 n+1) \Lambda(\lambda) /(2 \lambda+1)$.

In the sequel we will have occasion to investigate another constraint, viz., for $f \in F_{e} \cap C^{1}[-1,1]$ solve

$$
\min \left\|f-\phi_{2 n}\right\| \quad \text { subject to } \quad \phi_{2 n} \in \mathscr{P}_{2 n} \text { and } \dot{\phi}_{2 n}(1)=\dot{f}(1) \neq 0 \text {. }
$$


The reason why this particular constraint is of interest is the fact that the relative error at $x=1$ vanishes subject to the constraints given in (3.2).

For the problem stated in (3.2) we introduce

$$
\begin{aligned}
\Omega_{e}= & \int_{-1}^{1}\left(f(x)-\sum_{k=0}^{n} d_{k} C_{2 k, \lambda}(x)\right)^{2} w(x) d x+\mu \sum_{k=0}^{n} d_{k} C_{2 k, \lambda}(1) \\
& +\gamma\left\{\sum_{k=0}^{n} d_{k} \dot{C}_{2 k, \lambda}(1)-\dot{f}(1)\right\} .
\end{aligned}
$$

The condition $\partial \Omega_{e} / \partial d_{i}=0$ for $i=0,1, \ldots, n$, and the constraints yield the equations,

$$
\frac{\partial \Omega_{e}}{\partial d_{i}}=-2\left(f, C_{2 i, \lambda}\right)+2 h_{2 i} d_{i}+\mu C_{2 i, \lambda}(1)+\gamma \dot{C}_{2 i, \lambda}(1)=0
$$

and

$$
\begin{aligned}
& A(1,1) \frac{\mu}{2}+B(1,1) \frac{\gamma}{2}=(f, A(1, x)), \\
& B(1,1) \frac{\mu}{2}+C(1,1) \frac{\gamma}{2}=(f, B(1, x))-\dot{f}(1),
\end{aligned}
$$

where

$$
B(x, t)=\frac{\partial}{\partial t} A(x, t) \text { and } C(x, t)=\frac{\partial}{\partial x} B(x, t)=\frac{\partial^{2}}{\partial x \partial t} A(x, t) .
$$

From the lemma we get $B(1, t)=\dot{S}_{0}(t)=2(\lambda+1) \Lambda(\lambda) C_{2 n-1, \lambda+2}(t)$. In order to find the sum $B(x, 1)$, we may use the identity

$$
B(x, t)=q_{2 n+1} \frac{\partial}{\partial t}\left\{\frac{t C_{2 n+1, \lambda}(t) C_{2 n+2, \lambda}(x)-x C_{2 n+1, \lambda}(x) C_{2 n+2, \lambda}(t)}{x^{2}-t^{2}}\right\} .
$$

After much calculation we find

$$
B(x, 1)=\frac{4 \Lambda(\lambda)}{2 \lambda+1}\left((n+1)(\lambda+n+1) C_{2 n, \lambda+1}(x)-(\lambda+1) C_{2 n, \lambda+2}(x)\right) .
$$

However, we can obtain the same result without using the Christoffel-Darboux identity, by simply observing that

$$
B(x, 1)=\frac{4 \Lambda(\lambda)}{2 \lambda+1} \sum_{k=1}^{n} \frac{k(\lambda+k)}{\lambda}(2 k+\lambda) C_{2 k, \lambda}(x) .
$$

Using Eq. (1.1) and some manipulations we again obtain (3.3).

Differentiating (3.3) with respect to $x$ gives

$$
\begin{aligned}
C(x, 1)=\frac{8 \Lambda(\lambda)}{2 \lambda+1}((n+1)(\lambda+1)(\lambda & +n+1) C_{2 n-1, \lambda+2}(x) \\
& \left.-(\lambda+1)(\lambda+2) C_{2 n-1, \lambda+3}(x)\right) .
\end{aligned}
$$

4. Examples. As may be seen from Theorems 1 and 2 in Section 2, a main difficulty when calculating the least squares approximations is to achieve high-precision values of the inner products

$$
\gamma_{m, \lambda}(f)=\left(f, C_{m, \lambda}\right)=\int_{-1}^{1} f(x) C_{m, \lambda}(x)\left(1-x^{2}\right)^{\lambda-1 / 2} d x,
$$


where $m$ is even when $f \in F_{e}$ and odd when $f \in F_{o}$. Now, we give some ways for the calculation of the integral (4.1).

(a) Suppose that $f(x)$ is analytic and that $f \in F_{e}$. Then $f(x)$ may be expanded in a Fourier series of the form

$$
f(x)=\sum_{n=0}^{\infty} a_{2 n+1} \cos \left(\frac{2 n+1}{2} \pi x\right), \quad-1 \leqslant x \leqslant 1,
$$

and $\gamma_{2 m, \lambda}(f)$ written as

$$
\gamma_{2 m, \lambda}(f)=\sum_{n=0}^{\infty} a_{2 n+1} \int_{-1}^{1} C_{2 m, \lambda}(x) \cos \left(\frac{2 n+1}{2} \pi x\right)\left(1-x^{2}\right)^{\lambda-1 / 2} d x .
$$

Letting

$$
K_{m, \lambda}(a)=\int_{-1}^{1} C_{m, \lambda}(x) e^{i a x}\left(1-x^{2}\right)^{\lambda-1 / 2} d x
$$

and noting (see, e.g., Gradshteyn and Ryzhik [5, p. 830] or Erdélyi et al. [3, p. 178])

$$
K_{m, \lambda}(a)=i^{m} \frac{2 \pi \Gamma(2 \lambda+m)}{m ! \Gamma(\lambda)(2 a)^{\lambda}} J_{m+\lambda}(a), \quad \operatorname{Re} \lambda>-1 / 2,
$$

we obtain

$$
\gamma_{2 m, \lambda}(f)=\sum_{n=0}^{\infty} a_{2 n+1}(-1)^{m} \frac{2 \pi \Gamma(2 \lambda+2 m)}{(2 m) ! \Gamma(\lambda)((2 n+1) \pi)^{\lambda}} J_{2 m+\lambda}\left(\frac{2 n+1}{2} \pi\right) .
$$

The Bessel function $J_{2 m+\lambda}(a)$ may be computed by Miller's algorithm (see Abramowitz and Stegun [1, pp. 452-453]).

A similar formula may be obtained for $\gamma_{2 m+1, \lambda}(f)$ if $f \in F_{o}$ and $f$ is analytic.

(b) Quite another approach is the following. Let $f$ be analytic and belong to $F_{e}$. Then there exists an expansion of the form

$$
f(x)=\sum_{k=1}^{\infty} c(k)\left(1-x^{2}\right)^{k}, \quad x \in[-1,1] .
$$

Several different expressions for the coefficients $c(k)$ may be obtained similar to those given by Wrigge and Fransén [12, pp. 568-569]. Given the expansion (4.2), $\gamma_{2 m, \lambda}(f)$ becomes

$$
\gamma_{2 m, \lambda}(f)=\sum_{k=m}^{\infty} c(k) G_{2 m, k}(\lambda)
$$

where

$$
G_{2 m, k}(\lambda)=\int_{-1}^{1} C_{2 m, \lambda}(x)\left(1-x^{2}\right)^{k+\lambda-1 / 2} d x .
$$

A quick way to evaluate (4.3) is the following. We "invert" the relation (1.3) and write

$$
\left(1-x^{2}\right)^{k}=\sum_{n=0}^{k} \mu_{n, \lambda}(k) C_{2 n, \lambda}(x), \quad m \leqslant k .
$$

Then, because of orthogonality, we get

$$
G_{2 m, k}(\lambda)=\mu_{m, \lambda}(k)\left\|C_{2 m, \lambda}\right\|^{2}
$$


It is also possible to expand $C_{2 m, \lambda}(x)$ in powers of $x^{2}$ or $1-x^{2}$ and then evaluate the elementary integrals that arise. The formulae we then get are, however, much more complicated than (4.4).

It can be shown (see Apelblat [2, p. 189]) that

$$
G_{2 m, k}(\lambda)=(-1)^{m}\left(\begin{array}{c}
k \\
m
\end{array}\right) \frac{\sqrt{\pi} \Gamma(\lambda+m) \Gamma(\lambda+k+1 / 2)}{\Gamma(\lambda) \Gamma(\lambda+k+m+1)}, \quad m \leqslant k .
$$

Since the norm $\left\|C_{2 m, \lambda}\right\|$ is well-known, we have thus evaluated the coefficients $\mu_{m, \lambda}(k)$, which means that we have succeeded to explicitly invert the relation (1.3).

(c) An appropriate numerical method for the determination of the integrals $\gamma_{m, \lambda}(f)$ is the application of Gauss-Gegenbauer quadrature to (4.1). Error bounds for Gaussian quadrature of analytic functions were developed by Gautschi and Varga [4] (see also Smith [9]).

Example 4.1. $f(x)=\cos (\pi x / 2), x \in[-1,1]$. In this case we explicitly know that

$$
\int_{-1}^{1} C_{2 m, \lambda}(x) \cos (a x)\left(1-x^{2}\right)^{\lambda-1 / 2} d x=(-1)^{m} \frac{2 \pi \Gamma(2 \lambda+2 m)}{(2 m) ! \Gamma(\lambda)(2 a)^{\lambda}} J_{2 m+\lambda}(a) .
$$

Putting $a=\pi / 2$ and $\lambda=1 / 2$ we get $\gamma_{2 m, 1 / 2}(f)=2(-1)^{m} J_{2 m+1 / 2}(\pi / 2)$. Similarly, we find

$$
\begin{aligned}
\gamma_{0,0}(f) & =\left(f, T_{0}\right)=\pi J_{0}(\pi / 2), \\
\gamma_{2 m, 0}(f)=\left(f, T_{2 m}\right) & =2 \pi(-1)^{m} J_{2 m}(\pi / 2), \quad m>0 .
\end{aligned}
$$

These expressions are then used to calculate $d_{n, k}(1 / 2)$ and $d_{n, k}(0)$ [cf. Theorem 1].

The coefficients $d_{n, k}(0)$ for $n=1,2, \ldots, 10$ are presented in Table 4.1. The approximations for $\lambda=1 / 2$ are slightly less accurate than those for $\lambda=0$. The corresponding absolute errors

$$
\varepsilon_{n}(\lambda)=\max _{-1 \leqslant x \leqslant 1}\left|f(x)-\sum_{k=1}^{n} d_{n, k}(\lambda)\left(1-x^{2}\right)^{k}\right|,
$$

for $\lambda=0$ and $\lambda=1 / 2$, are displayed also in Table 4.1. Numbers in parentheses indicate decimal exponents.

Example 4.2. $f(x)=J_{0}\left(a_{0} x\right), a_{0}=\left\{\min x>0: J_{0}(x)=0\right\}$. Accurate values of $a_{0}$ and more generally $a_{\nu}$, where $a_{\nu}=\left\{\min x>0: J_{\nu}(x)=0\right\}$, are given to 100D by Zironi [13, p. 335]. We have, e.g., $a_{0}=2.40482555769577276862 \ldots$.. We consider especially the case $\lambda=0$, because in Gradshteyn and Ryzhik [5, p. 836] we find the prerequisite result

$$
\int_{0}^{1}\left(1-x^{2}\right)^{-1 / 2} T_{n}(x) J_{\nu}(a x) d x=\frac{\pi}{2} J_{(\nu+n) / 2}(a / 2) J_{(\nu-n) / 2}(a / 2),
$$

$a>0, \operatorname{Re} \nu>-n-1$.

For $\nu=0$ and $n=2 k$ we have

$$
\int_{-1}^{1}\left(1-x^{2}\right)^{-1 / 2} J_{0}(a x) T_{2 k}(x) d x=\pi(-1)^{k} J_{k}^{2}(a / 2) .
$$

The coefficients $d_{n, k}(0), n=1,2, \ldots, 10$, in the approximation

$$
J_{0}\left(a_{0} x\right) \cong \sum_{k=1}^{n} d_{n, k}(0)\left(1-x^{2}\right)^{k}, \quad x \in[-1,1]
$$


TABLE 4.1

\begin{tabular}{|c|c|c|c|c|}
\hline$n$ & k & $d_{n, k}(0)$ & $\varepsilon_{n}(0)$ & $\varepsilon_{n}(1 / 2)$ \\
\hline 1 & 1 & 0.962270459871251081530834 & $3.83(-2)$ & $4.06(-2)$ \\
\hline 2 & $\begin{array}{l}1 \\
2\end{array}$ & $\begin{array}{l}0.777230028061934424939665 \\
0.222048518171179987909402\end{array}$ & $7.46(-4)$ & $8.88(-4)$ \\
\hline 3 & $\begin{array}{l}1 \\
2 \\
3\end{array}$ & $\begin{array}{l}0.785557128488924080168619 \\
0.195401796804813091176750 \\
0.019033372404547783380466\end{array}$ & $8.05(-6)$ & $1.06(-5)$ \\
\hline 4 & $\begin{array}{l}1 \\
2 \\
3 \\
4\end{array}$ & $\begin{array}{l}0.785396470018426704804616 \\
0.196365747627797343360769 \\
0.017380885279431922493576 \\
0.000856845175986001941350\end{array}$ & $5.44(-8)$ & $7.78(-8)$ \\
\hline 5 & $\begin{array}{l}1 \\
2 \\
3 \\
4 \\
5\end{array}$ & $\begin{array}{l}0.785398174745279410389985 \\
0.196349382250011369741228 \\
0.017429981412789843352201 \\
0.000798657166080317960758 \\
0.000023804185870507082970\end{array}$ & $2.49(-10)$ & $3.90(-10)$ \\
\hline 6 & $\begin{array}{l}1 \\
2 \\
3 \\
4 \\
5 \\
6\end{array}$ & $\begin{array}{l}0.785398163345269475623525 \\
0.196349541850150456471664 \\
0.017429251812154018298777 \\
0.000800116367351968067604 \\
0.000022477639259916076746 \\
0.000000448985006661571337\end{array}$ & $8.52(-13)$ & $1.39(-12)$ \\
\hline 7 & $\begin{array}{l}1 \\
2 \\
3 \\
4 \\
5 \\
6 \\
7\end{array}$ & $\begin{array}{l}0.785398163397623018186441 \\
0.196349540844962439263683 \\
0.017429258274076986064370 \\
0.000800097220913545058441 \\
0.000022506358917550590491 \\
0.000000427776644100699649 \\
0.000000006126860295362932\end{array}$ & $2.07(-15)$ & $3.74(-15)$ \\
\hline 8 & $\begin{array}{l}1 \\
2 \\
3 \\
4 \\
5 \\
6 \\
7 \\
8\end{array}$ & $\begin{array}{l}0.785398163397447864822406 \\
0.196349540849376304037355 \\
0.017429258236243859432897 \\
0.000800097375048505408886 \\
0.000022506022623091644066 \\
0.000000428180197451435359 \\
0.000000005875760432682935 \\
0.000000000063302486389915\end{array}$ & $4.33(-18)$ & $7.60(-18)$ \\
\hline 9 & $\begin{array}{l}1 \\
2 \\
3 \\
4 \\
5 \\
6 \\
7 \\
8 \\
9\end{array}$ & $\begin{array}{l}0.785398163397448310505497 \\
0.196349540849362042178425 \\
0.017429258236400739881121 \\
0.000800097374211809685025 \\
0.000022506025095147191835 \\
0.000000428175937909568433 \\
0.000000005880019974549860 \\
0.000000000061011640343838 \\
0.000000000000512426089254\end{array}$ & $7.09(-21)$ & $1.35(-20)$ \\
\hline 10 & $\begin{array}{r}1 \\
2 \\
3 \\
4 \\
5 \\
6 \\
7 \\
8 \\
9 \\
10\end{array}$ & $\begin{array}{l}0.785398163397448309614225 \\
0.196349540849362077472803 \\
0.017429258236400255843973 \\
0.000800097374215072453947 \\
0.000022506025082689346862 \\
0.000000428175966658441448 \\
0.000000005879979087263795 \\
0.000000000061046686589037 \\
0.000000000000495825236265 \\
0.00000000000003337737638\end{array}$ & $8.99(-24)$ & $1.89(-23)$ \\
\hline
\end{tabular}


are calculated. The corresponding absolute errors are given by $6.97(-2), 2.70(-3)$, $6.10(-5), 8.83(-7), 8.90(-9), 6.58(-11), 3.73(-13), 1.65(-15), 6.00(-18)$, and 2.53(-20), respectively. For example, if $n=10$ we have the following coefficients $c(k)=d_{10, k}(0)$ :

$$
\begin{array}{ll}
c(1)=0.624229584847753322534, & c(6)=0.000014291669769704969, \\
c(2)=0.312114792423876793755, & c(7)=0.000000433105085872767, \\
c(3)=0.057658371084764508079, & c(8)=0.000000009988565281248, \\
c(4)=0.005639239014138741338, & c(9)=0.000000000180796461075, \\
c(5)=0.000343277682412430896, & c(10)=0.000000000002836883322 .
\end{array}
$$

We also note the following general expansion (see, e.g., Magnus et al. [6, p. 125]),

$$
J_{\nu}(x z)=x^{\nu} \sum_{n=0}^{\infty}\left(\frac{z}{2}\left(1-x^{2}\right)\right)^{n} J_{\nu+n}(z)(n !)^{-1} \text {. }
$$

Of special interest is, of course, the case $\nu=0$ and $z=a_{0}$. For computational purposes we also considered the expansion

$$
J_{2 m}\left(a_{2 m} x\right)=\sum_{k=1}^{\infty} C_{2 m}(k)\left(1-x^{2}\right)^{k}, \quad m=0,1,2, \ldots
$$

Using the differential equation for $J_{2 m}(z)$ we could prove that the coefficients $C_{2 m}(k)$ satisfy the recurrence relation

$$
\left\{\begin{array}{l}
4(k+2)(k+1) C_{2 m}(k+2)-4(k+1)(2 k+1) C_{2 m}(k+1) \\
+\left(4 k^{2}+a_{2 m}^{2}-4 m^{2}\right) C_{2 m}(k)-a_{2 m}^{2} C_{2 m}(k-1)=0, \quad k=2,3,4, \ldots
\end{array}\right.
$$

Starting values are given by

$$
\begin{gathered}
C_{2 m}(1)=\frac{1}{2} a_{2 m} J_{2 m+1}\left(a_{2 m}\right), \quad C_{2 m}(2)=\frac{1}{2} C_{2 m}(1), \\
C_{2 m}(3)=C_{2 m}(2)-\frac{4+a_{2 m}^{2}-4 m^{2}}{24} C_{2 m}(1) .
\end{gathered}
$$

Since (4.6), (4.7) is ill-conditioned as it stands, we had to rewrite it and could then use Miller's algorithm. We thus calculated $C_{0}(k)$ and $C_{2}(k)$ for $k=1,2, \ldots, 70$. The convergence was very fast.

Example 4.3. In this example we consider $f(x)=\cos (\pi x / 2)$ with the extra constraint $\phi_{2 n}(0)=1$. As weight function we choose $w(x)=\left(1-x^{2}\right)^{-1 / 2}$, i.e., $\lambda=0$. We then approximate $\cos (\pi x / 2)$ in $L_{2}$-norm (with the mentioned weight function) by a polynomial of the form

$$
\cos (\pi x / 2) \cong \sum_{k=1}^{n} d_{n, k}\left(1-x^{2}\right)^{k}, \quad x \in[-1,1]
$$

where the coefficients satisfy the constraint

$$
\sum_{k=1}^{n} d_{n, k}=1
$$

We notice that $f(1-2 x)=\cos (\pi(1-2 x) / 2)=\sin (\pi x)$, where $x \in[0,1]$. The corresponding approximations for $\sin (\pi x)$ are then of the form

$$
\sin (\pi x) \cong \sum_{k=1}^{n} d_{n, k^{4}} 4^{k}(x(1-x))^{k}
$$


Using the result in Section 3, we obtain after much calculation

$$
d_{n, k}=(-4)^{k} \sum_{i=k}^{n} \frac{i}{i+k}\left(\begin{array}{c}
i+k \\
2 k
\end{array}\right) a_{i}^{(n)}, \quad k=1,2, \ldots, n,
$$

where

$$
\begin{aligned}
a_{0}^{(n)} & =J_{0}(\pi / 2)-A_{n}, \\
a_{2 i}^{(n)} & =2\left(J_{4 i}(\pi / 2)-A_{n}\right), \quad i=1,2, \ldots, \\
a_{2 i-1}^{(n)} & =-2\left(J_{4 i-2}(\pi / 2)+B_{n}\right), \quad i=1,2, \ldots .
\end{aligned}
$$

The coefficients $A_{n}$ and $B_{n}$ are given by

$$
A_{n}=\frac{2 n+1-(-1)^{n}}{4 n(n+1)}\left(2 J_{0}(\pi / 2)+4 S_{n}-1\right)
$$

and

$$
B_{n}=\frac{2 n+1+(-1)^{n}}{4 n(n+1)}\left(1-4 U_{n}\right)
$$

where

$$
S_{1}=0, \quad S_{n}=\sum_{i=1}^{[n / 2]} J_{4 i}(\pi / 2), \quad U_{n}=\sum_{i=1}^{[(n+1) / 2]} J_{4 i-2}(\pi / 2) .
$$

$J_{n}$, of course, denotes the Bessel function of order $n$.

The coefficients $d_{n, k}, n=1,2, \ldots, 10$, are calculated. The corresponding absolute errors are given by $5.60(-2), 9.25(-4), 9.36(-6), 6.12(-8), 2.78(-10), 9.23(-13)$, $2.34(-15), 4.66(-18), 7.50(-21)$, and $9.91(-24)$, respectively.

All calculations were performed in $Q$-arithmetic on a VAX $11 / 780$ computer at the Institute of Earthquake Engineering and Engineering Seismology, University "Kiril and Metodij" Skoplje.

Acknowledgment. The authors are grateful to the referees and to Professor Walter Gautschi for helpful suggestions and comments, and to Mrs. K. Maneva for help in programming.

Faculty of Electronic Engineering

Department of Mathematics

University of Niš

P. O. Box 73

18000 Niš, Yugoslavia

National Defence Research Institute

Division I, Section 123

Box 27322

S-102 54 Stockholm, Sweden

1. M. Abramowitz \& I. A. Stegun, Handbook of Mathematical Functions with Formulas, Graphs, and Mathematical Tables, Dover, New York, 1965.

2. A. Apelblat, Tables of Definite and Infinite Integrals, Elsevier, Amsterdam-Oxford-New York, 1983.

3. A. Erdélyi et al., Higher Transcendental Functions, Vol. II, McGraw-Hill, New York, 1953.

4. W. Gautschi \& R. S. VARga, "Error bounds for Gaussian quadrature of analytic functions," SIAM J. Numer. Anal., v. 20, 1983, pp. 1170-1186.

5. I. S. Gradshteyn \& I. M. RyzhiK, Tables of Integrals, Series, and Products, Academic Press, New York, 1980. 
6. W. Magnus, F. Oberhettinger \& R. P. Soni, Formulas and Theorems for the Special Functions of Mathematical Physics, Springer-Verlag, Berlin and New York, 1966.

7. B. P. Rakovich \& P. M. Vasic, "Some new sums for orthogonal polynomials involved in filter synthesis," Univ. Beograd. Publ. Elektrotehn. Fak. Ser. Mat. Fiz., No. 544-No. 576, 1976, pp. 8-12.

8. T. J. Riviln, An Introduction to the Approximation of Functions, Dover, New York, 1981.

9. H. V. SмIтH, “Global error bounds for Gauss-Gegenbauer quadrature," BIT, v. 21, 1981, pp. 481-490.

10. G. Szego, Orthogonal Polynomials, Amer. Math. Soc. Colloq. Publ., vol. 23, Amer. Math. Soc., Providence, R. I., 1939.

11. S. Wrigge, A General Method of Approximation Associated with Bernstein Polynomials, FOA Rapport, C 10170-M8, December 1980, National Defence Research Institute, S-10450 Stockholm, Sweden.

12. S. Wrigge \& A. Fransen, “A general method of approximation. Part I,” Math. Comp., v. 38, 1982, pp. $567-588$

13. F. ZIRONI, "Multiple precision computation of some zeros of Bessel functions by rigorous explicit formulae," Calcolo, v. 18, 1981, pp. 321-335. 